\title{
Investigating the History of Magmatic Volatiles in the Moon Using NanoSIMS
}

\author{
Jessica J. Barnes ${ }^{1}$, Mahesh Anand ${ }^{1,2}$, Ian A. Franchi ${ }^{1}$. \\ 1. Planetary \& Space Science, The Open University, Walton Hall, Milton Keynes, UK \\ 2. Department of Earth Sciences, The Natural History Museum, Cromwell Road, London, UK
}

For decades the Moon was considered an anhydrous planetary body, significantly depleted in volatiles, including water, compared to the Earth [1]. It wasn't until recently that water $\left(\mathrm{H}_{2} \mathrm{O}\right.$ equivalent) and other volatiles were confidently measured in a variety of lunar samples (e.g., [2-5]), particularly in lunar volcanic glass beads, melt inclusions, and apatite $\left[\mathrm{Ca}_{5}\left(\mathrm{PO}_{4}\right)_{3}(\mathrm{~F}, \mathrm{Cl}, \mathrm{OH})\right]$ from samples returned by the Apollo missions. The Earth and Moon are isotopic twins in many respects (e.g., [6-7]), however, they have very different chlorine isotopic compositions [8-9]. [The chlorine isotopic composition is reported in permil using a delta notation where: $\delta^{37} \mathrm{Cl}(\%)=\left({ }^{37} \mathrm{Cl} /{ }^{35} \mathrm{Cl}\right.$ sample $/{ }^{37} \mathrm{Cl} /{ }^{35} \mathrm{Cl}$ standard $)-1 \times 1000$, where the standard ratio is relative to standard mean ocean chloride $\left(\mathrm{SMOC},{ }^{37} \mathrm{Cl} /{ }^{35} \mathrm{Cl}\right.$ ratio $\left.\left.\left.=0.31977\right]\right)\right]$. When compared to terrestrial rocks and chondritic (carbonaceous and ordinary) meteorites $\left(\delta^{37} \mathrm{Cl} \sim 0 \%\right.$, e.g., [10]), lunar samples show highly variable chlorine isotopic compositions ranging from $\sim-4$ to $+24 \%$ [8-9]. The cause(s) for this heavy chlorine isotopic signature is most likely related to magmatic degassing (loss) of light ${ }^{35} \mathrm{Cl}$ in metal chlorides, a process that would enrich the remaining melt in ${ }^{37} \mathrm{Cl}$ [8]. However it remains unclear at what stages(s) in the Moon's history such fractionation(s) may have occurred.

The focus of our work has been on lunar apatite, since it is the most common hydrous mineral in a variety of lunar rock types, permitting investigations of the evolution of lunar volatiles over geological time (from $\sim 4.5$ to 3 billion years ago). In order to fully understand the history of volatiles in the Moon, it is necessary to have the capability to make high precision, in situ measurements of chlorine isotopes in apatite crystals that range in size between $\sim 5$ and $300 \mu \mathrm{m}$ in length. It is also important to be able to determine the abundance of the other volatiles ( $\mathrm{F}$ and $\mathrm{H}$, as $\mathrm{OH}$ ) occupying the same $\mathrm{X}$ site in the apatite crystal structure, so that apatite volatile chemistry and crystal stoichiometry can be assessed. Achieving both of these goals simultaneously is a challenging task given the range in masses of interest.

A large suite of lunar samples, provided as polished thin sections by the NASA's Curation and Analysis Planning Team for Extraterrestrial Materials (CAPTEM) and the Meteorite Working Group, were investigated in this study. Samples were characterized by both optical and scanning electron microscopy. Phosphates were identified as P-rich areas in X-ray maps and apatite was positively identified using low current $(0.6 \mathrm{nA})$ energy dispersive spectroscopy. Each apatite crystal was imaged at high resolution using both back scattered electron and secondary electron techniques, the former to characterize the surrounding mineralogy and petrographic setting of apatite and the latter to ensure the sample surface was suitable for subsequent analysis by ion probe (Fig. 1). The Cameca Nano Secondary Ion Mass Spectrometer (NanoSIMS 50L) instrument at The Open University was used to analyze the samples. NanoSIMS boasts exceptional spatial resolution that allows an assessment of the presence of any volatile heterogeneities through multiple spot analyses on individual apatite crystals. The ion probe was operated in $\mathrm{Cs}^{+}$mode and all negative secondary ions $\left({ }^{16} \mathrm{OH},{ }^{18} \mathrm{O},{ }^{19} \mathrm{~F},{ }^{35} \mathrm{Cl}\right.$, and $\left.{ }^{37} \mathrm{Cl}\right)$ were collected simultaneously on electron multipliers. We have developed a protocol to precisely measure the isotopes of $\mathrm{Cl}\left({ }^{35} \mathrm{Cl}\right.$ and $\left.{ }^{37} \mathrm{Cl}\right)$ along with $\mathrm{OH}, \mathrm{Cl}, \mathrm{F}$ abundances (Fig. 2) using relatively low probe currents of generally 25 to $60 \mathrm{pA}$ [11]. Typically areas of between $3 \times 3$ and $5 \times 5 \mu \mathrm{m}$ were analyzed, depending on the crystal size and the requirement to avoid cracks or inclusions. The reproducibility of ${ }^{37} \mathrm{Cl} /{ }^{35} \mathrm{Cl}$ ratio of the apatite standards is typically better than $\sim 1.5$ $\%$ [11]. 
Our latest work includes analyses of lunar apatite from Apollo mare basalt and highlands (norite and troctolite) samples and lunar basaltic meteorites. We have found that in general apatite from highlands rocks and incompatible element-rich basalts have elevated $\delta^{37} \mathrm{Cl}$ values of between $\sim+15$ and $+36 \%$ and $\mathrm{Cl}$ contents in the range of 0.3 to $1.4 \mathrm{wt}$ \% [11]. In contrast, apatite from incompatible element-poor basalts have $\mathrm{Cl}$ isotopic compositions in the range of +2 to $+16 \%$ with $\mathrm{Cl}$ contents from $\sim 0.03$ to $\sim 0.9$ wt. \%. Based on recent studies investigating the abundance and isotopic composition of water in the Moon it is likely that water inside the Moon shares an affinity with water found in the Earth's mantle [12-14], carbonaceous chondrites [12-14] and the HED parent body 4Vesta [15]. Whilst the Moon and Earth seem to share a common history with regards to the accretion of water, when compared to the Earth, Mars and asteroids, the Moon shows highly fractionated chlorine isotope compositions [8-9, 11]. Because we have measured a geochemically varied suite of samples we are able to show that the Cl-isotopic composition recorded by apatite is related to the bulk rare earth element ratios of the rocks [11]. Our working hypothesis to reconcile these $\mathrm{Cl}$ isotope data involves processes occurring during the primary differentiation of the Moon and earliest degassing/volatile loss history of the Moon, not experienced by Earth [11]. [16]

\section{References:}

[1] Taylor, S. R. et al, Rev. Mineral. Geochem. 60 (2006), 657-704.

[2] Saal, A. E. et al, Nature 454 (2008), 192-195.

[3] McCubbin, F. M. et al, PNAS 27 (2010), 11223-11228.

[4] Hauri, E. et al, Science 333 (2011), 213-215.

[5] Greenwood, J. P. et al, Nat. Geosci., 4 (2011), 79-82.

[6] Wiechert, U. et al, Science 294 (2001), 345-348.

[7] Armytage, R. M. G. et al, Geochim. Cosmochim. Acta 77 (2012), 504-514.

[8] Sharp, Z. D. et al, Science 329 (2010), 1050-1053.

[9] Boyce, J. W. et al, Sci. Adv. 1 (2015), e1500380.

[10] Sharp, Z. D. et al, Geochim. Cosmochim. Acta 107 (2013), 189-204.

[11] Barnes, J. J. et al, In review in Earth Planet. Sci. Lett.

[12] Saal, A. E. et al, Science 340 (2013), 1317-1320.

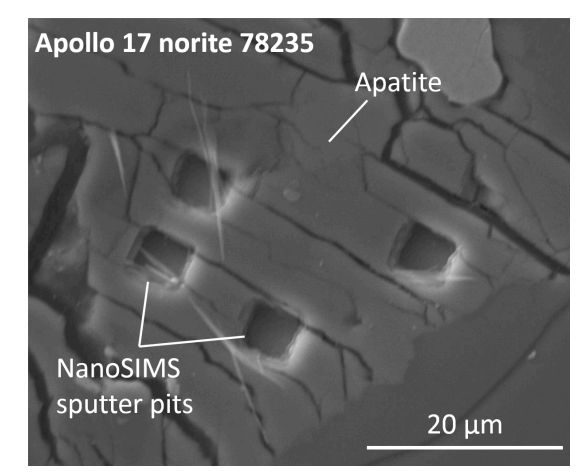

Figure 1. (Left) Secondary electron image showing the typical NanoSIMS sputter pit sizes and precision positioning required for analyzing extraterrestrial samples. Figure 2. (Right) Secondary ion [13] Tartèse, R. et al, Geology 42 (2014), 363-366. [14] Barnes, J. J. et al, Earth Planet. Sci. Lett. 390 (2014), 244-252. [15] Sarafian, A. et al, Science 346 (2014), 623-626.

[16] We acknowledge funding from STFC, UK and thank Mineralogical Society of Great Britain and Northern Ireland for an award with which $\mathrm{JJB}$ is able to attend this meeting.

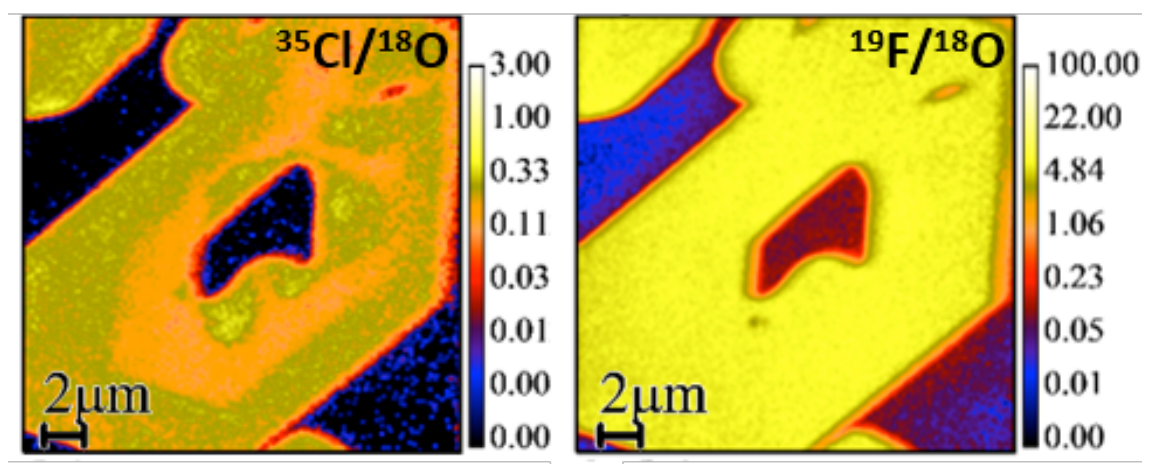

images (log scale) showing the relative amounts of $\mathrm{F}$ and $\mathrm{Cl}$ in apatite (yellow) and glass (blue-black) in a mare basalt (12039). 